\title{
NAISSANCE ET DEVELOPPEMENT DE LA CARTE DU CIEL EN FRANCE
}

\author{
Théo WEIMER \\ Observatoire de Paris \\ 61 avenue de 1 'Observatoire \\ 75014 Paris \\ France
}

ABSTRACT. This is a summary of the publication "Brève histoire de 1a Carte du Ciel en France". Th. Weimer, Observatoire de Paris Pub., 1987.

En 1839 les premières photographies, obtenues par Niepce et Daguerre, furent présentées à 1 'Académie des Sciences de Paris. Elles étaient obtenues par l'action de la lumière sur une couche d'iodure d'argent. Ce procédé était peu pratique pour des applications scientifiques ; néanmoins, il a permis d'obtenir des daguerréotypes de la Lune (Draper, 1840) et du Soleil (Fizeau et Foucault, 1845). D'autres essais furent faits ; mais il a fallu la mise au point des plaques au gélatino-bromure d'argent pour penser à des applications astronomiques plus systématiques.

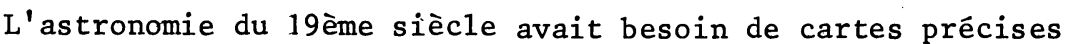
du ciel pour repérer et situer planètes nouvelles et comètes. A 1 'Observatoire de Paris, on avait entrepris de dessiner les cartes des régions écliptiques. Mais les observations devenaient presque impraticables dans les régions galactiques. Pickering aux Etats-Unis, David Gill en Afrique du Sud songèrent, dès 1882 , à la photographie pour de telles cartes. L'Amiral Mouchez, Directeur de l'Observatoire de Paris, en présentant à 1 'Académie des Sciences des photographies obtenues par D. Gil1, reprit 1 'idée à son compte.

Les Frères Paul et Prosper Henry, astronomes à l'Observatoire de Paris, mirent 1 'idée en pratique : un objectif de $16 \mathrm{~cm}$ de diamètre, spécialement taillé, leur donna en octobre 1984 le premier cliché de 1 'Amas de Persée. Soutenus par Mouchez, ils réalisèrent, en 1885, avec le concours de $P$. Gautier pour la mécanique un instrument plus important $\left(33 \mathrm{~cm}\right.$ d'ouverture, $343 \mathrm{~cm}$ de distance focale). C'est $1^{\prime}$ ancêtre et le prototype des équatoriaux de la Carte du Ciel. 
L'Amiral Mouchez pensa aussitôt à une coopération pour "1a construction d'une carte du ciel par la photographie" et, avant même de lancer, par l'intermédiaire de l'Académie des Sciences, une invitation à une cinquantaine de savants de toutes nationalités, il avait obtenu des crédits pour trois équatoriaux destinés aux observatoires français.

La réunion de cette première conférence eut lieu à Paris du 16 au 27 avril 1887. C'était une préfiguration de 1'UAI (à part le nombre de participants!) : discours et réceptions par les personnalités politiques et scientifiques, mais aussi, et surtout, beaucoup de discussions et de travail dans des commissions spécialisées. Tout était à créer : caractéristiques des instruments, méthode d'obtention des clichés, leur mesure, leur réduction, conservation des documents pour 1 'avenir.

Dix-huit observatoires, répartis dans le monde entier, participèrent à l'entreprise. Mais il a fallu une nombreuse correspondance et des décisions du Comité international permanent de 1889 et de 189] pour clarifier les idées et codifier les décisions : Le travail se fera avec un réfracteur à deux verres, achromatisé pour le bleu, de $33 \mathrm{~cm}$ d'ouverture, $343 \mathrm{~cm}$ de focale (1' correspond à $1 \mathrm{~mm}$ sur le cliché) et un champ de $2^{\bullet} \times 2^{\bullet}$; on fera deux séries de clichés comportant plusieurs poses, pour distinguer étoiles et faux points ; 1'une pour faire un catalogue d'étoiles jusqu'à la magnitude 11, 1'autre pour une carte jusqu'à la magnitude 14 ; afin de contrôler d'éventuelles déformations de la gélatine et pour faciliter les mesures on copiera sur chaque cliché 1 'image d'un réseau à mailles de $5 \mathrm{~mm}$ de côté. D'autres recommandations, sur les méthodes de mesures et de calcul, sur la reproduction des cartes, furent faites ; mais elles n'avaient pas de caractère impératif.

Le travail était lancé et de nouvelles conférences eurent lieu en $1896,1900,1909$ pour préciser certains points (système d'étoiles de repère, etc.). La Guerre de 1914-1918 et ses conséquences économiques avaient entraîné des retards, mais aussi la mise en place de nouvelles organisations, création de 1 'Union Astronomique Internationale, dont 1a Commission 23, "Carte du Ciel", prit la suite des structures précédentes. E1le disparut à son tour en 1970, 1orsque 1 'on estima que les buts fixés en 1887 par les fondateurs avaient été atteints, Son travail n'a pas été vain et le Centre de Données Stellaires de Strasbourg, en particulier, poursuit par des moyens modernes les buts initiaux. Surtout n'oublions pas que la conférence de 1887 a jeté les bases de la coopération internationale dans la science, et a introduit dans l'astronomie l'emploi de la photographie. 


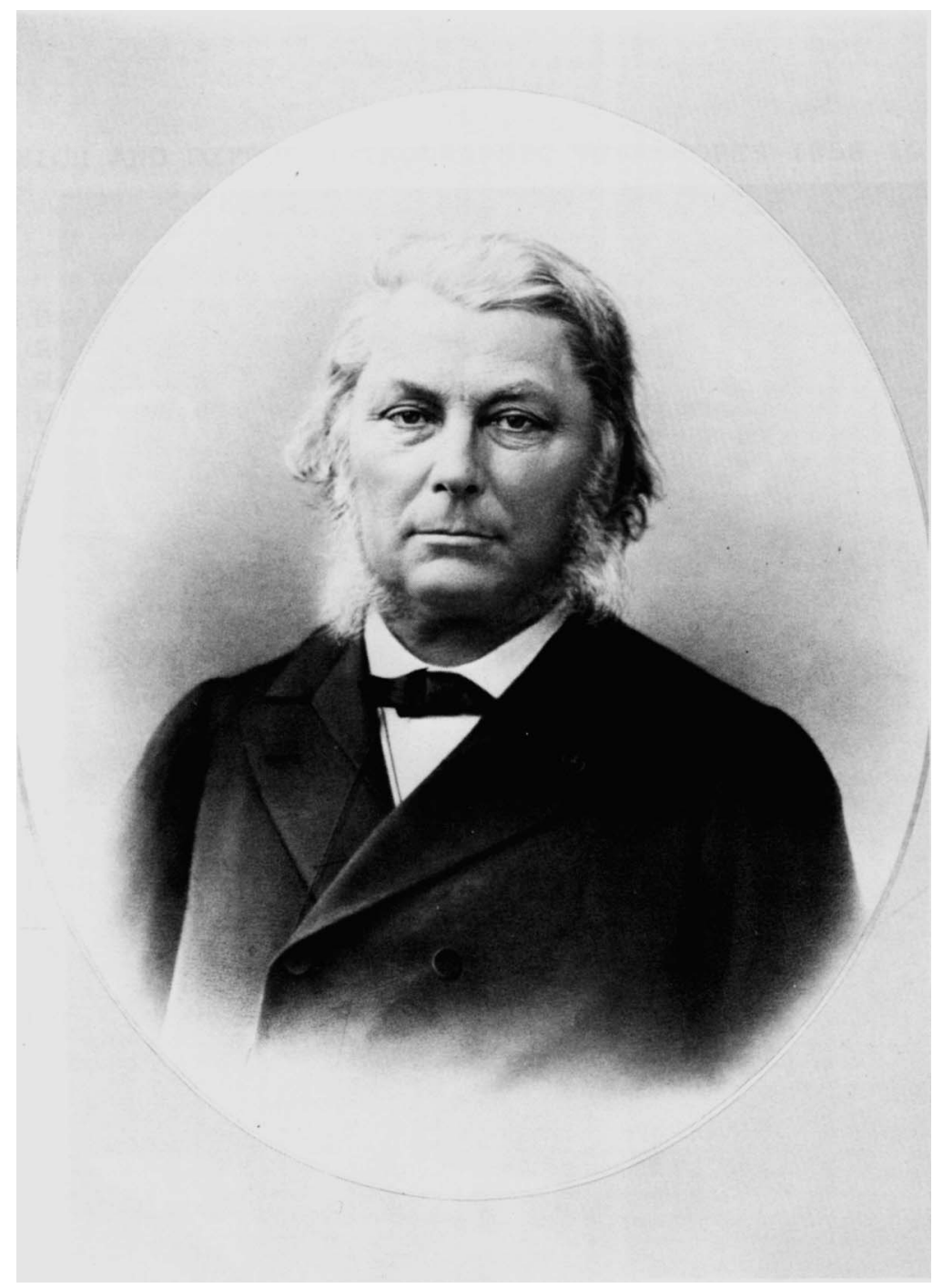

Figure 1: L'Amiral Mouchez (1821 - 1892)

Collection Bibliothèque de l'Observatoire de Paris 


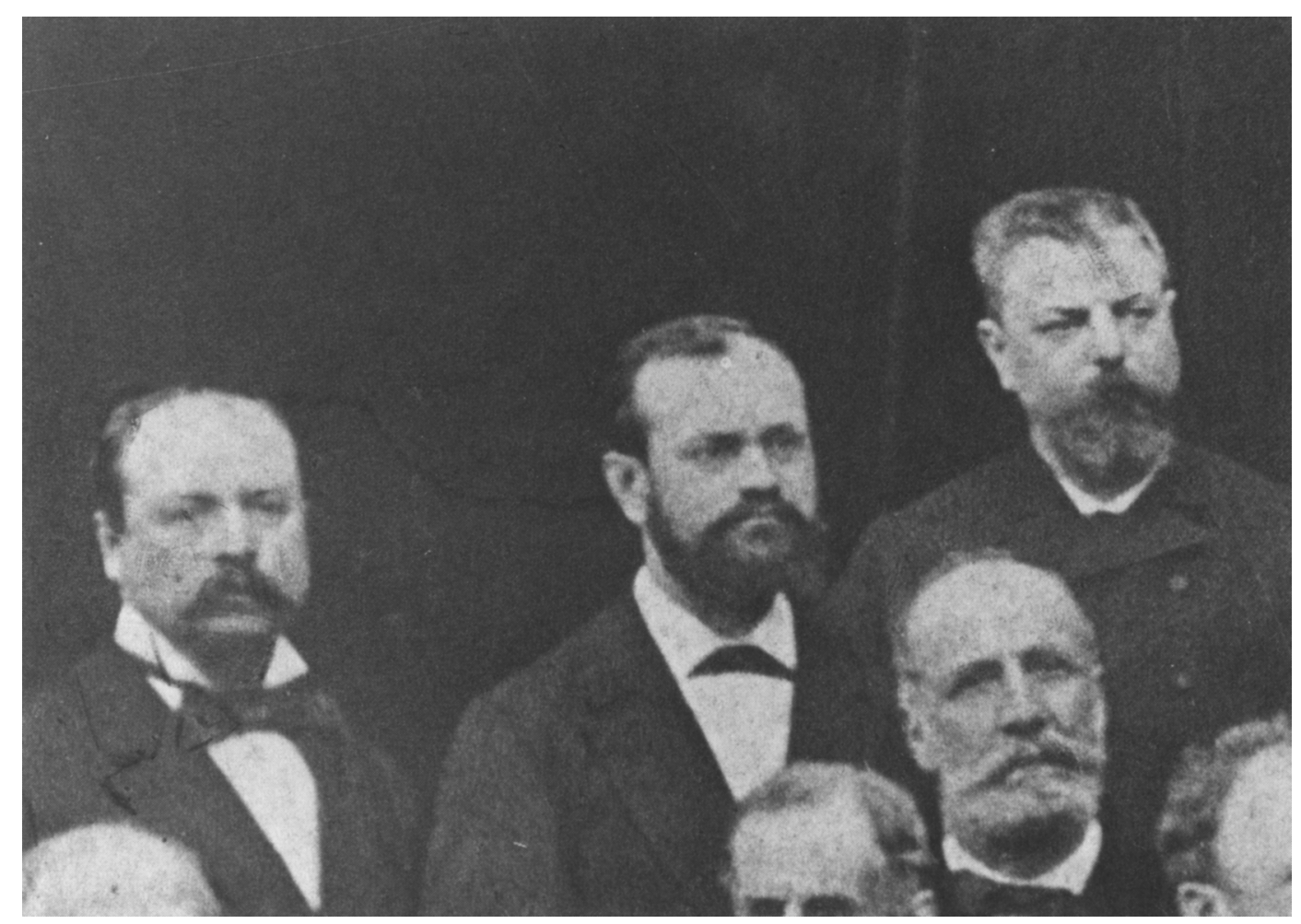

Figure 2: Les Frères Henry (de gauche à droite, Paul et Prosper) et P. Gautier Extrait d'un cliché Nadar - Collection Bibliothèque de 1'Observatoire de Paris 